La

Révolution

française

\section{La Révolution française}

Cahiers de l'Institut d'histoire de la Révolution française

$3 \mid 2012$

Les comités des assemblées révolutionnaires : des laboratoires de la loi

\title{
Le Comité des Inspecteurs de la salle : une institution au service de la Convention nationale (1792-1795)
}

The Committe of the Inspecteurs de la Salle: an institution at the service of the Convention

Alain Cohen

\section{OpenEdition}

Journals

Édition électronique

URL : http://journals.openedition.org//rf/755

DOI : $10.4000 / / r f .755$

ISSN : 2105-2557

Éditeur

IHMC - Institut d'histoire moderne et contemporaine (UMR 8066)

Référence électronique

Alain Cohen, «Le Comité des Inspecteurs de la salle : une institution au service de la Convention nationale (1792-1795) », La Révolution française [En ligne], 3 | 2012, mis en ligne le 20 décembre 2012, consulté le 01 mai 2019. URL : http://journals.openedition.org/Irf/755 ; DOI : 10.4000/Irf.755

Ce document a été généré automatiquement le 1 mai 2019.

(c) La Révolution française 


\section{Le Comité des Inspecteurs de la salle : une institution au service de la Convention nationale (1792-1795)}

The Committe of the Inspecteurs de la Salle: an institution at the service of the Convention

\section{Alain Cohen}

1 L'organisation interne de l'Assemblée ne date pas de la Convention nationale puisque le premier règlement, adopté par la Constituante, est du 29 juillet 1789. Il n'accordait qu'une place restreinte aux comités. Les Constituants français se sont inspirés de la Chambre anglaise des Communes, dont les comités se sont imposés en raison de la nécessité technique d'organiser le travail des parlementaires. Cet exemple n'a pas manqué d'exercer une influence sur les Constituants français lorsqu'ils voulurent créer le premier comité de constitution. Comme la Constituante faisait également œuvre de législation courante, il fallait diviser le travail de préparation des très nombreux textes entre des comités rassemblant des spécialistes du droit dans les domaines suivants: législation criminelle, défense nationale, finances, questions sociales, droits de l'homme, questions coloniales etc.........Au total, ce furent une vingtaine de comités permanents qui virent le jour au cours de la première année de la Révolution. Chaque comité préparait le travail de l'Assemblée en lui présentant des rapports et des projets de décret. Mais, c'était l'Assemblée plénière qui prenait seule les décisions par l'adoption des décrets, sur propositions de chaque comité en fonction de sa compétence. Comme le précise André Castaldo $^{1}$ à propos de la Constituante, les comités ont participé à la «mission constitutionnelle et législative de l'Assemblée [...] ces organes ont souvent et en même temps des responsabilités qui, normalement, sont dévolues à un exécutif ou même à un pouvoir judiciaire ». L'augmentation considérable du travail parlementaire entraîna un accroissement parallèle des effectifs de l'administration de l'Assemblée, illustré par le nombre de commis qui atteignit la centaine en avril 1790. Ces faits expliquent l'origine des Commissaires et Inspecteurs de la salle. 


\section{L'ébauche d'une organisation des Inspecteurs du local de la salle (Constituante et Législative)}

2 Lorsque l'Assemblée siégeait à Versailles (jusqu'au 18 octobre 1789) les dépenses de la salle dépendaient du Ministère de la Maison du Roi et du budget qui lui était affecté. Cette situation perdura jusqu'au 1er octobre 1790, alors que l'Assemblée décida de transporter ses services de la salle de l'Archevêché de Paris (après le 7 novembre 1789) au Manège des Tuileries. La voie était toute tracée pour l'autonomie administrative et financière de l'Assemblée par rapport à la Maison du roi. La désignation du député Guillotin comme Commissaire de la salle jeta les bases de l'administration de l'Assemblée puisqu'elle le chargea de veiller sur la police, la sûreté et la salubrité de la salle. Les termes « d'inspecteurs des dépenses des bureaux des comités » ou "d'inspecteurs du travail des commis » apparurent alors parce qu'il s'agissait de permettre à Guillotin d'être secondé. Mais, ces inspecteurs n'étaient pas encore organisés en comité sous la Constituante et la Législative. Ils ne le furent qu'à partir de la Convention nationale. Les commis furent placés sous l'autorité de deux, puis quatre députés élus par leurs pairs en qualité d'Inspecteurs. Selon un décret du 23 octobre 1789, deux membres de l'Assemblée devaient se déplacer dans les bureaux afin de «s'assurer de la manière dont les commis rempliront leurs devoirs et même de l'utilité ou nécessité de l'emploi. Les plaintes, que les membres de l'Assemblée auraient à faire contre les commis, ainsi que celles que les commis auraient à faire les uns contre les autres, seront adressées aux inspecteurs $»^{2}$. Ces derniers avaient des fonctions très larges, autant administratives que techniques et financières en rapport avec le fonctionnement d'une assemblée qui se développa fortement au fur et à mesure que l'exécutif perdait peu à peu de son importance. Les exemples abondent, comme par exemple quand il fallut transformer à partir de novembre 1789 les locaux vétustes du Manège des Tuileries (2 monastères, celui des Capucines et celui des Feuillants) en salles et bureaux d'assemblée. L'Assemblée décida alors de nommer cinq Commissaires pour aider Guillotin à contrôler ces travaux (députés d'Aiguillon, Gouy, La Poule, Le Pelletier de Saint-Fargeau, Colbert de Castlehill). Les inspecteurs de la salle passaient commande des fournitures (chandelles, bougies y compris le petit matériel) et assuraient le contrôle de l'Imprimerie nationale en payant à Baudouin, son Directeur, les frais d'impression des procès-verbaux de l'assemblée, ainsi que des décrets, rapports, tableaux des finances, affiches et opinions particulières des représentants du peuple. En outre, ce furent les inspecteurs qui réglementèrent l'occupation privative de certains espaces situés sur le domaine public de l'assemblée (contrôle des travaux faits par les occupants, baux, négociations avec d'éventuels propriétaires privés). Les inspecteurs devaient veiller sur la sécurité des locaux d'autant plus que des accidents pouvaient se produire, comme ce fut le cas le 26 octobre 1789 dans les locaux de l'Archevêché de Paris lors : «Une tribune s'écroula : quatre représentants du peuple furent blessés, ainsi que plusieurs spectateurs $»^{3}$. Autre attribution essentielle des inspecteurs : le contrôle des travaux immobiliers nécessités par la maintenance, voire la construction d'extensions des locaux de l'assemblée. Enfin, les inspecteurs furent chargés de surveiller l'évolution des dépenses du personnel employé par les différents comités.

3 Ce découpage des missions incombant aux inspecteurs ne fut pas fondamentalement modifié sous la Convention nationale. Mais, les interventions de ceux-ci se 
systématisèrent au fur et à mesure de l'extension du rôle de la Convention nationale et de son emprise de plus en plus déterminante sur les destinées du pays.

\section{L'amplification des compétences ou attributions des Inspecteurs sous la Convention}

4 En effet, ces compétences ou attributions s'étoffèrent sous la Convention par rapport à celles déjà définies par l'Assemblée nationale de juillet 1789 à septembre 1792 et s'étendirent à de nouveaux domaines confiés par la Convention nationale en rapport avec la montée en puissance de l'Assemblée par rapport au pouvoir exécutif.

\section{Gestion et contrôle des travaux immobiliers : cause première du rôle croissant du comité}

Comme nous le verrons à propos des contacts avec le Ministère de l'Intérieur, le Comité des Inspecteurs de la salle n'assumait pas la responsabilité de la maîtrise d'ouvrage des travaux immobiliers puisque le Ministère de l'Intérieur, héritier direct de la Maison du roi, conserva cette attribution jusqu'à sa disparition (1er avril 1794), notamment pour la construction et l'aménagement des salles de séances de l'Assemblée. Le Comité des Inspecteurs de la Salle intervenait plutôt en position de contrôleur des travaux (à la fois financier et technique) dans la mesure où il devait donner son avis sur le déblocage des fonds pour l'aménagement des locaux des comités de la Convention ${ }^{4}$. En ce qui concerne l'aménagement des espaces communs du Palais, l'architecte rattaché au Comité des Inspecteurs autorisait la construction des échoppes sous les arcades de la galerie, après approbation des plans par le comité5. Pour exercer ces responsabilités techniques, le Comité des Inspecteurs s'appuyait sur la compétence d'hommes de l'art, comme l'inspecteur général Vacquer et l'architecte Gisors sans lesquels « nul travail ne pourra être entrepris par les entrepreneurs et ouvriers sans un ordre de l'Architecte et de l'Inspecteur général des travaux ${ }^{6} »$.Le comité, aidé également de ses experts vérificateurs comme Bimulec, se prononçait sur les réclamations des entreprises en matière de révision de prix ou d'appréciation du prix en lui-même ${ }^{7}$. Ces interventions à caractère technique étaient souvent accompagnées d'appréciations juridiques, comme cela a été relevé en matière de gestion du patrimoine immobilier de la Convention nationale.

\section{Le comité au service de la Convention pour gérer son patrimoine immobilier}

6 Les espaces libres, corridors ou couloirs du Palais national pouvaient être occupés privativement par des commerçants ou particuliers qui sollicitaient l'accord de la Convention sur la signature de leurs baux. Celle-ci chargeait le Comité des Inspecteurs d'examiner ces demandes. Les locations pouvaient être régularisées ou au contraire résiliées, selon une appréciation juridique qui relevait de la compétence des juristes du comité. Les critères retenus par celui-ci pouvaient être politiques, comme l'exclusion d'un bénéfice énorme au moyen d'une sous-location totale de locaux révélatrice d'un esprit de spéculation ${ }^{8}$, par opposition à des locations de petits lots à des particuliers pères de famille dont la petite propriété devait être encouragée. Les résiliations de bail pouvaient 
être aussi prononcées par le comité qui devait, en ce cas, apprécier si des indemnités compensatrices de perte de jouissance devaient ou non être accordées aux locataires évincés9.

7 La prévention des accidents entrait dans les compétences du Comité des Inspecteurs. Il en fut ainsi à la veille d'une manifestation publique à l'édifice de l'Opéra. À la demande du Comité de salut public, en sa séance du 18 germinal an II-7 avril 1794, le Comité des Inspecteurs désigna deux de ses membres, assistés d'architectes, d'un maçon et d'un charpentier, pour s'assurer que " cet édifice est assez solide pour accueillir le peuple qui doit y affluer avec abondance $\aleph^{10}$. Ces mesures de prévention revêtaient un caractère permanent puisque l'inspecteur des bâtiments, Lecomte, et l'architecte Gisors devaient visiter «chaque matin et soir toutes les dépendances et les maisons où sont établis les comités de la Convention depuis les toits jusqu'aux fondations pour s'assurer de leur état et faire rapport au Comité $»^{11}$. Cette vigilance devait être efficace puisque fut constaté un sinistre conduisant à une reconstruction. Les attributions du Comité des Inspecteurs ne se limitaient pas aux questions immobilières et portaient également sur l'administration générale, c'est-à-dire les frais de personnel, dépenses d'énergie, fournitures diverses et frais de mission des représentants du peuple.

\section{Le comité assure l'administration générale de la Convention nationale}

8 Le Comité des Inspecteurs devait remettre régulièrement à la Convention un état des appointements annuels de l'ensemble de ses employés. En suivant les recommandations de la Convention, il s'efforça d'appliquer une politique d'harmonisation des traitements des employés de la salle et des comités, ce qui ne fut pas du goût de ces derniers. Après Thermidor an II, une nouvelle organisation des comités se mit en place par laquelle ces derniers eurent le droit d'autoriser les dépenses nécessaires à l'organisation de leurs bureaux et de fixer un niveau de traitements de leurs commis correspondant à leurs compétences. Des augmentations provisoires de traitements en résultèrent, si bien que le Comité des finances enjoignit au Comité des Inspecteurs de les approuver ${ }^{12}$. Les employés de la salle présentèrent alors des demandes d'augmentations de leurs traitements, à telle enseigne que le Comité des Inspecteurs prit un arrêté fixant une grille salariale par catégorie de personnel (2000 livres pour les premiers garçons; 1800 livres pour les deuxièmes garçons ; 1650 livres pour les garçons de la salle ; 1800 livres pour les premiers lustriers; 1650 livres pour les autres lustriers). La politique salariale de la Convention nationale fut avantageuse pour les commis qualifiés et cadres, sauf pour les garçons de bureaux et chefs de division, ce qu'indique également Catherine Kawa pour le personnel du ministère de l'Intérieur ${ }^{13}$.

9 Le Comité des Inspecteurs intervint dans d'autres questions que celles afférentes aux traitements des agents et commis. La Convention faisait une consommation extrêmement élevée de fluides (bois de chauffage, cire pour les bougies par exemple) en raison, bien sûr, des séances continuelles de nuit des députés et membres des comités. Le comité chargea l'un de ses membres, le représentant du peuple Robin, « de l'établissement d'un rapport général sur le mode le plus économique d'illuminer la salle et les bureaux de la Convention ». Comme la consommation des chandelles augmentait journellement et que l'approvisionnement de la Convention « ne peut se faire sans prendre sur les besoins les plus urgents du peuple », il fut question de faire usage de bougies ou d'huile pour éclairer 
les bureaux et « en surveillant très strictement la consommation et en ne permettant pas qu'on en abuse $\aleph^{14}$. Le Comité des Inspecteurs intervint comme un ministère technique pour faciliter l'approvisionnement en cire indispensable à la fabrication des bougies. Il mit aussi à contribution la Commission du commerce et des approvisionnements pour obtenir le bois nécessaire au chauffage de la Convention, sans toutefois "prendre sur les besoins urgents du peuple pour faire arriver chaque décade dans les fourrières du Palais et de ses comités la quantité indispensable à sa consommation $»^{15}$.

Le Comité des Inspecteurs pouvait intervenir de manière assez inattendue, par exemple, pour fournir aux représentants du peuple des costumes pour les cérémonies auxquelles ils devaient participer. Certains corps détachés auprès de la Convention bénéficièrent de meubles et de linge qui leur étaient attribués par les Inspecteurs de la salle, comme ce fut le cas des vétérans invalides affectés à des tâches de police au sein du Palais et du Jardin national (séance du Comité des Inspecteurs du 23 frimaire an II-13 décembre 1793) ${ }^{16}$. Le Comité des Inspecteurs fut aussi chargé de vérifier les comptes des frais de voyage des représentants du peuple envoyés en mission dans les départements et aux armées (cf. loi du 7 fructidor an II-24 août 1794). Il eut beaucoup de mal à exercer ce contrôle surtout à partir des derniers mois de l'année 1795, d'autant plus que le Comité des Inspecteurs disparut avec la Convention nationale (26 octobre 1795). En effet, il était difficile pour lui de censurer les dépenses de représentants en mission. Par contre, le contrôle hiérarchique du personnel de la Convention était davantage dans les possibilités du comité.

\section{Le comité est-il le chef du personnel de la Convention?}

11 Le rattachement des employés de la salle allait de soi puisque les inspecteurs de la salle étaient les chefs hiérarchiques des commis, garçons de salles, huissiers. Il en allait différemment des employés dépendant des comités. Mais, c'était par le biais de l'ameublement des comités que les inspecteurs pouvaient exercer la surveillance et la responsabilité du personnel des comités qui avait la garde des meubles et effets. C'était donc par souci de cohérence que le Règlement de l'Assemblée attribuait au Comité des Inspecteurs la nomination des garçons de salle et des bureaux (séances du Comité des Inspecteurs du 28 septembre 1793 et du 23 frimaire an II-13 décembre 1793) ${ }^{17}$. En outre, l'établissement des états des appointements des employés de la Convention, confié à l'Inspection, jouait dans le sens de la création d'un bloc de compétences au profit du Comité des Inspecteurs, à l'exception toutefois de la fixation des traitements que les comités entendaient conserver résolument. La gestion des effectifs des employés de la Convention par le Comité des Inspecteurs portait sur les affectations et mutations. L'action de ce dernier s'apparentait à celle d'une direction du personnel. Il devait aussi veiller au respect des décisions prises par les comités et pouvait ainsi assurer la discipline au sein de l'administration des bureaux de la Convention, exigeant le respect de la morale républicaine, d'autant plus que les fonctionnaires publics non élus et les employés payés par les deniers de la République étaient tenus de justifier de l'obtention d'un certificat de civisme $^{18}$.

12 Le Comité des Inspecteurs vit ses responsabilités s'accroître dans de fortes proportions au fur et à mesure de l'emprise croissante de la Convention nationale sur la vie politique et économique du pays. L'autonomie de cette dernière ne pouvait être sauvegardée que si son territoire était protégé, voire demeure inviolé face aux menaces et attaques 
éventuelles de l'extérieur. D'où les attributions conférées au Comité des Inspecteurs en matière de sécurité de la Convention, de contrôle politique et militaire du Palais et du Jardin national, de maintien de l'ordre à l'intérieur de l'espace de la Convention et de ses abords immédiats.

\section{La sécurité intérieure et extérieure du Palais et du Jardin national : les attributions militaires et politiques du comité}

13 La garde des entrées du Palais et du Jardin national était assurée par des gendarmes et la garde nationale. Le Comité des Inspecteurs eut l'idée de renforcer cette protection par l'affectation d'un nouveau corps composé de militaires invalides. Au départ, ce dernier était placé sous la surveillance conjointe du ministère de l'Intérieur et du Comité des Inspecteurs. Un changement se produisit avec l'effacement du ministère de l'Intérieur, si bien que le ministre de la Guerre remplaça ce dernier. Les abus de comportement de certains militaires aboutirent à la suppression du corps des invalides, à la suite d'une concertation entre le Comité militaire et le Comité des Inspecteurs ${ }^{19}$.

La police des tribunes, des couloirs et corridors du Palais national était d'une importance essentielle, même si les citoyens pouvaient accéder aux salles de la Convention, au moins en théorie. Toutefois, les cartes d'accès aux tribunes étaient très réglementées, ce qui n'empêchait pas les abus d'utilisation par des particuliers des cartes de représentants du peuple. D'où l'intervention du Comité des Inspecteurs pour exiger le renouvellement des cartes de représentants du peuple et des secrétaires ${ }^{20}$. Il accorda aussi des laissez-passer aux ministres de la République et fit délivrer des cartes d'accès aux ministres des cours étrangères et alliées de la République. Si des désordres se produisaient au sein des tribunes, les huissiers déféraient les auteurs d'incidents ou d'actes de violence devant le Comité des Inspecteurs qui pouvait les faire conduire auprès du Commissaire de police en vue de leur arrestation ${ }^{21}$. Le Jardin national avait été également le théâtre de manifestations politiques les 1er et 2 prairial an III-20 et 21 mai 1795. En effet, la Convention fut envahie en ces journées par la foule, ce qui permit aux députés de la Montagne de voter, dans la confusion, quelques décrets favorables aux revendications populaires. Après que la Convention eut repris le contrôle de la salle des séances, le Comité des Inspecteurs proposa à cette dernière un décret, daté du 6 prairial an III-25 mai 1795, qui prévoyait l'application de mesures préventives pour empêcher le passage des « factieux » par les issues particulières des traiteurs débouchant sur le Jardin national. Le 19 messidor an III-7juillet 1795, soit un mois et demi après les incidents, le Comité des Inspecteurs adressa une lettre au Comité de salut public pour insister sur la fermeture de ces issues privées ouvertes la nuit «aux femmes de mauvaise vie qui, même dans la journée, donnent le spectacle le plus outrageant aux bonnes mœurs ${ }^{22}$.

Le Comité des Inspecteurs exerçait aussi des attributions militaires en demandant au Commandant de la Garde nationale de prendre ses ordres directement auprès de lui. Il désignait les emplacements qui devaient être gardés par les factionnaires ou sentinelles aux entrées du Palais et du Jardin national. Il fut amené à prendre lui-même directement des mesures militaires pour prévenir une action contre la Convention. C'est ainsi qu'il apprit que plusieurs pièces de canon avaient été placées aux entrées du Palais et du Jardin des Tuileries. Après enquête demandée par le Comité des Inspecteurs, il apparut que ces canons avaient été placés sur un ordre du général Mathis. Le comité rappela dans une lettre adressée au Commandant général qu' « il ne doit y avoir aucune force armée dans 
l'enceinte de la Convention sans un décret qui n'en émane et une réquisition particulière de son Comité des Inspecteurs de la salle». Dans une lettre du comité du 24 juillet 1793, celui-ci réaffirma ses responsabilités militaires; mais, en l'occurrence, il ne s'agissait que d'une simple bavure militaire sans conséquence ${ }^{23}$.

Le Comité des Inspecteurs avait aussi des relations avec la police parisienne, lorsque des vols étaient commis en dehors du Palais national, notamment à l'encontre des fédérés de passage à Paris. Le comité autorisa les membres de la police parisienne à requérir la force armée «sauf à eux à rendre compte au comité de leurs opérations de ce jour jusqu'au départ des fédérés ${ }^{24}$. Les instructions données par le Comité des Inspecteurs aux administrateurs de police de la Commune de Paris n'étaient pas limitées aux délits ou crimes commis aux abords du Palais et du Jardin national. En effet, le Comité des Inspecteurs avait compétence pour obtenir de la police parisienne la liste nominative des représentants du peuple en état d'arrestation et notamment l'identité des maisons d'arrêt dans lesquelles ils étaient détenus ${ }^{25}$. Le comité n'exerçait pas seulement de compétences relevant de la police ou de l'emploi des forces armées puisqu'il coopérait également à l'application de la politique culturelle définie par la Convention nationale, en liaison avec le Comité de salut public.

\section{Une politique prestigieuse et ambitieuse d'entretien et d'embellissement du Palais et du Jardin national}

Suivant les instructions du Comité de salut public, les Inspecteurs apportèrent leur décisive contribution à la conservation des monuments publics. Ainsi, le Comité des Inspecteurs ordonna que « les bustes, dessins et estampes offerts aux assemblées soient retirés des archives pour être placés dans le salon de la Liberté dans la mesure où ils sont jugés dignes d'être exposés ${ }^{26}$. Cette association du Comité des Inspecteurs à la mise en place de la politique culturelle du gouvernement révolutionnaire allait au-delà de la conservation des monuments publics puisque le comité intervint aussi pour organiser un concours destiné aux artistes et gens de lettres pour donner aux « barrières de Paris » le caractère d'un monument public en vue de retracer les époques révolutionnaires et les victoires des armées de la République ${ }^{27}$. Le Comité des Inspecteurs pouvait aussi participer aux opérations d'embellissement du Palais et du Jardin national, comme ce fut le cas pour le plan de ce projet défini par le Comité de salut public (avant thermidor an II). Mais, après l'élimination du groupe de Robespierre, le Comité des Inspecteurs imprima sa marque au projet en élaguant le plan initial du Comité de salut public qu'il considérait comme trop onéreux ${ }^{28}$.

Dans le domaine de la préparation des fêtes, il faut signaler aussi la contribution du Comité des Inspecteurs à la préparation de la fête du 10 août (1795), comme la construction d'un orchestre pour la célébration du 3ème anniversaire de la suspension de Louis XVI. Quand il s'agissait d'assurer la protection d'un concert dans le Jardin national, le Comité des Inspecteurs prit les mesures de police en désignant les effectifs à placer dans cet espace.

Ce rôle aussi riche incombant au Comité des Inspecteurs s'amplifia encore avec la mise en œuvre de la politique du gouvernement révolutionnaire durant la Terreur. 


\section{Les biens d'Église : leur recensement par le comité. Un rôle essentiel pour le comité}

Il pouvait paraître étonnant que le comité jouât un rôle aussi essentiel dans un tel domaine aussi éloigné de l'administration générale de la Convention. Ce fait s'explique par des considérations tirées du rôle stratégique incombant au Comité de salut public en matière de politique religieuse et économique. Le Comité des Inspecteurs devint pendant la Terreur le fidèle exécutant du Comité de salut public et bien sûr de la Convention. Les séances du Comité des Inspecteurs du 24 et 26 brumaire an II-14 et 16 novembre 1793 nous apportent tous éclaircissements sur le rôle majeur qui revint au Comité des Inspecteurs dans l'application de cette politique religieuse et économique définie par la Convention. Le comité décida de constituer avec d'autres comités une commission chargée de procéder au tri des matières déposées provenant des dépouilles de l'Église et de « distraire des matières d'or, d'argent et de cuivre offertes à la patrie à partir des ouvrages précieux qui dépendent de ces matières $»^{29}$. Le Comité des Inspecteurs devint à cette occasion un organisme coordonnateur et centralisateur de ces opérations amorcées par les communes qui «drainaient les dépouilles» de l'Église jusqu'à Paris où intervenaient les représentants de la Commission des monuments, la Trésorerie nationale, de la Monnaie et de l'administration des domaines nationaux. Le Comité des Inspecteurs garda la haute main sur les opérations de tri, de recollement et de vérification de ces biens précieux. Mais, la gestion des biens de l'Église n'était pas le seul domaine stratégique d'action des Inspecteurs dans la mesure où l'Imprimerie nationale était dès la Constituante l'attribution clé du Comité.

\section{L'Imprimerie nationale : un secteur politiquement très sensible. Le contrôle du comité sur les réquisitions et approvisionnements en papier}

21 Le Comité des Inspecteurs intervenait en ce domaine parce qu'il était l'interlocuteur naturel de l'Imprimerie nationale qui avait succédé à l'Imprimerie royale. C'était ce comité qui devait s'assurer de l'impression des lois et procès-verbaux de la Chambre et donc ensuite de la Convention. La politique des prix d'impression incombait au comité. Il renforça ses structures en faisant appel à des experts vérificateurs des impressions de l'Imprimerie nationale. Il soumit aussi celle-ci à l'aiguillon de la concurrence, comme l'atteste la séance du Comité des Inspecteurs du 6 thermidor an III-24 juillet $1795^{30}$. Les attributions du comité s'accrurent dans de très fortes proportions parallèlement au développement de la politique économique dirigiste, que ce soit à propos des augmentations de salaires des ouvriers ou pour les approvisionnements de l'Imprimerie nationale en papiers. Sur le premier point, la séance extraordinaire du Comité des Inspecteurs du 4 ventôse an II-22 février 1794 nous apprend que les ouvriers de l'Imprimerie nationale demandèrent une augmentation des salaires (sur la base du maximum). Pour enrayer les mouvements sociaux, comme les abandons de poste ou les grèves perlées, le comité demanda une enquête à deux de ses inspecteurs Fiquet et Duval ${ }^{31}$. Ensuite, il décida de renouveler la mise en réquisition des manufactures et exigea le transfert des demandes d'augmentations de salaires, soit à la Convention, soit au Comité 
des Inspecteurs, soit enfin aux commissaires placés par ce dernier auprès de ces manufactures.

Sur le deuxième point, c'est-à-dire des approvisionnements des imprimeries en papiers, le comité appliqua une politique de réquisition en employant, dans certains cas, des fournisseurs privés de papiers pour les réquisitions de papiers ou, dans d'autres cas, en envoyant en mission un de ses inspecteurs (Chabanon) chez les fabricants et possesseurs de papiers (en Haute Vienne ou au Puy-de-Dôme) ${ }^{32}$.

Les réquisitions de matières premières ne furent pas limitées aux papiers nécessaires à l'Imprimerie nationale. Elles pouvaient également porter sur du suif, du charbon, des huiles, de la cire destinée aux bougies. Indépendamment de ce rôle économique et social, le Comité des Inspecteurs amplifia ses responsabilités politiques liées à l'expression des opinions.

\section{Le contrôle du comité sur les expressions des opinions politiques}

Comme il a été indiqué plus haut, le comité eut un rôle clé dans le domaine de la diffusion des textes officiels. Par une pente en quelque sorte naturelle, il devait assurer aussi la diffusion des rapports ou mémoires en défense des représentants du peuple. Nous avons plus haut qu'il s'était attaché à connaître les noms des représentants qui avaient fait l'objet d'arrestation. Il était tout à fait logique qu'il contribuât à la diffusion de leurs écrits servant à leur défense devant les tribunaux, dans la mesure, toutefois, où il obtenait l'autorisation par la Convention de faire imprimer aux frais de la République le nombre d'exemplaires du mémoire ou du rapport. Le cas le plus fameux est fourni par la défense $\mathrm{du}$ représentant du peuple, Jean-Baptiste Carrier $^{33}$. Le comité dut prendre ses responsabilités politiques et se prononça, dans des circonstances délicates, pour la Montagne, même si ses membres appartenaient plutôt au courant de la Plaine.

Ainsi, le Comité des Inspecteurs intervint dans de nombreux domaines en fonction des besoins de la Convention dont il était un fidèle exécutant. Il convient à présent de connaître son organisation et sa composition.

\section{Composition du Comité des Inspecteurs : les modalités de sa désignation et de son organisation}

26 La séance du comité en date du 25 octobre 1792 indique sa dénomination à savoir: « Comité des Inspecteurs de la salle, des bureaux et de l'Imprimerie de la Convention ». Ce titre annonce déjà son rôle. Son organisation se mit en place sous forme de sections. Par ailleurs, les conditions de son élection et de son renouvellement se précisèrent peu à peu.

\section{Élection et renouvellement des Inspecteurs de la salle : un recrutement purement interne}

Comme nous l'avons vu en première partie, il est apparu que dès la Constituante les Inspecteurs étaient choisis au sein de l'Assemblée et élus par leurs pairs puisque cette dernière ne voulut pas déléguer ses attributions à des personnes extérieures à son instance. La Convention nationale ne souhaita pas modifier ce système. Il y eut donc continuité sur ce plan entre les assemblées. La période charnière se situa avec la montée 
en puissance du Comité de salut public à partir de son renouvellement intervenu le 10 juillet 1793 qui correspondit à la non réélection de Danton. Il y a donc lieu de diviser cette période en deux séquences : la première antérieure à la désignation du Grand Comité de salut public et la seconde qui prend effet du 10 juillet 1793 jusqu'au 9 thermidor an II-27 juillet 1794.

Dès le 25 octobre 1792, il fut décidé que tous les membres des comités feraient l'objet d'un renouvellement tous les mois, notamment les présidents et secrétaires. Lors de sa séance du 29 décembre 1792, le Comité des Inspecteurs confirma le renouvellement par moitié de ses membres au moyen d'une " liste par tirage au sort qui sera distribuée par ses soins aux deux bouts de la salle et une autre sera distribuée où chacun s'inscrirait pour le Comité où il voudra rentrer ». Donc, le Comité des Inspecteurs organisa non seulement le renouvellement de ses membres, mais aussi les scrutins pour les élections aux différents comités $^{34}$. Le principe retenu par l'Assemblée reposa sur les élections des différents représentants du peuple qui, après leur non renouvellement dans un comité, s'inscrivaient sur des listes pour être élus comme membres d'autres comités, ce qui permettait un brassage intéressant pour se former aux différentes matières et disciplines traitées par la Convention.

Il existait toutefois une limite à ce renouvellement des comités, selon une disposition du règlement adopté en juillet 1789, interdisant à un représentant du peuple d'être membre de deux comités à la fois, disposition guère respectée. Mais, le renouvellement par tirage au sort des membres du Comité des Inspecteurs revêtait un caractère automatique, selon un décret de l'Assemblée de 1793, disposant que les membres « qui sont restés en dernier tirage sont ceux qui, de plein droit, doivent quitter leurs fonctions $\aleph^{35}$. Le comité, en sa séance du 8 juin 1793, précisait que les autres membres devaient rester en fonction parce qu'ils étaient chargés de la signature, comme Saurine, Fiquet, Huguet, Projean, Guillemardet, Louis. Il semble qu'il y ait eu une certaine permanence de certains postes, mais aussi un renouvellement pour permettre le départ en mission de certains membres du Comité des Inspecteurs, ce qui présentait l'avantage, pour ces derniers, d'être confrontés aux réalités d'un terrain pour le moins mouvant. Mais, les «comités de gouvernement ", comme le Comité de salut public, exercèrent une influence certaine sur le fonctionnement des comités, spécialement à partir du Grand Comité de salut public.

Après la non réélection de Danton, il fut procédé au renouvellement du Comité de salut public, et la Convention décida le 13 septembre 1793 de renouveler tous les comités, dont le Comité des Inspecteurs. À partir du lendemain, le Comité de salut public était autorisé par la Convention à lui soumettre les listes des députés candidats aux fonctions de membres des comités. Ce pouvoir était évidemment décisif pour l'élection de membres dont les positions politiques étaient proches des Montagnards (comme Couthon et SaintJust), même si au sein du Comité des Inspecteurs les députés de la Plaine étaient nombreux, comme Blondel, Bouchereau, Bourgeois, Chabanon, Chedaneau, Chiappe, Colaud La Salcette, Debry, Duval de l'Aube, Fiquet, Herard, Martinel et Servière, soit la majorité des inspecteurs.

31 Un procès-verbal du Comité des Inspecteurs, en sa séance du 8 octobre 1793, illustra cette pratique politique. Ce comité demanda au Comité de salut public de présenter à la Convention la liste des membres qui devaient composer le Comité des Inspecteurs, compte tenu du remplacement nécessaire de Saurine, girondin, mis en état d'arrestation, et alors qu'il devenait urgent de compléter le comité réduit à un trop petit nombre ${ }^{36}$. 
32 Après le 9 thermidor an II, la loi du 7 fructidor de la même année procéda à une réorganisation des comités par laquelle le Comité de salut public perdit le droit de soumettre à la Convention les listes de membres proposés pour les comités. En vertu de la même loi, le nombre des membres des comités devait être réduit, ce qui obligea le Comité des Inspecteurs à procéder au renouvellement de ses membres tous les mois, par tirage au sort de quatre membres. La description des règles d'élection et de renouvellement des mandats de membres du Comité des Inspecteurs n'est pas suffisante pour comprendre son fonctionnement. D'où la nécessité de connaître les règles qui président à son organisation interne.

\section{Organisation interne du Comité des Inspecteurs : une division des tâches en rapport avec l'extension de ses missions}

L'organisation du Comité des Inspecteurs s'étoffa avec l'accroissement de ses effectifs, qui fut très élevé durant la Terreur, en raison notamment de l'importance des attributions économiques qui lui furent conférées par l'Assemblée pour organiser la répartition des « dépouilles » de l'Église et aussi mettre en œuvre les réquisitions de matières premières, notamment de papier. Pour comprendre l'évolution de cette organisation, deux périodes doivent être distinguées, la première comprise entre la fin de l'année 1792 et qui court jusqu'à la fin du mois de mai 1794 et la seconde de juin 1794 à la fin du mois d'octobre 1795.

Au 25 octobre 1792, le Comité des Inspecteurs fut divisé en trois sections : la première des inspecteurs de la salle proprement dite, la seconde correspondant au secrétariat et la troisième, celle de l'imprimerie. Cette organisation était assez schématique dans la mesure où la première section était trop générale, alors que les inspecteurs exerçaient déjà des fonctions très spécialisées. Le comité le comprit, en sa séance du 9 prairial an II-28 mai $1794^{37}$, puisqu'il adopta une organisation beaucoup plus élaborée.

En effet, le comité reposait désormais sur cinq sections : la première, dont les membres étaient Robin, Chabanon et Bouchereau, portait sur le papier d'impression et les fournitures; la seconde confiée à Debry et Projean intervenait pour la surveillance et l'examen des comptes de l'Imprimerie nationale, ainsi que pour les bureaux de distribution ; la troisième, dont les membres étaient Huguet et Armonville, était chargée de la salle, des forces de sécurité, des vétérans, des gendarmes et enfin des consignes ; la quatrième section, composée de Duval, d'Herard, Chedaneau, Fiquet, intervenait pour les mandats et l'examen des comptes de dépenses; enfin la cinquième confiée à Sergent et Finol était en charge des travaux publics, des emplacements des comités et des boutiques. Le comité ajoutait que les membres attachés à chaque section pouvaient être changés tous les trois mois, ce qui ne pouvait que favoriser les rotations et éviter une trop grande spécialisation.

36 À la fin de l'année 1794, un renouvellement fut opéré avec l'arrivée de nouveaux inspecteurs spécialisés. D'où une nouvelle organisation arrêtée par le comité, en sa séance du 29 frimaire an III-19 décembre 1794, qui retint une nouvelle division en cinq sections. La première, confiée à Huguet, Bouchereau, Colaud et Chabanon, avait en charge l'approvisionnement en papier et autres fournitures diverses et les mémoires à régler ; la seconde, assurée par Rouzet, Couturier et Bougoin, avait en charge la surveillance et l'examen des comptes de l'Imprimerie nationale ; la troisième, représentée par Martinel, Duval et Belin, exerçait les fonctions suivantes : police de la salle, secrétariat, inspection 
des comités, consignes, surveillance des divers postes de l'Assemblée, à savoir: grenadiers, vétérans invalides, garde nationale, emplacements des comités ; la quatrième, confiée à Chedaneau, Fiquet, Hérard et Albert, avait en charge l'examen des comptes de toutes les dépenses et la surveillance de tous mandats à délivrer ; quant à la cinquième, elle était assurée par Poultier et Gantois, intervenant pour les travaux publics de tous les ouvrages de la Convention et les embellissements du Jardin national ${ }^{38}$.

Les archives nationales (surtout la série D) contiennent peu de renseignements sur les effectifs contrôlés par les inspecteurs, à l'exception toutefois des huissiers. Déjà sous la Constituante, les requêtes conservées par les Archives nationales nous renseignent sur la motivation des huissiers qui s'adressèrent aux Commissaires et Inspecteurs pour souligner le "rôle actif et intime » qu'ils assuraient auprès des députés lors des séances et les fonctions qu'ils exerçaient. Ces éléments étaient, selon ces huissiers, de nature à justifier « une distinction qui semble tenir à la dignité de MM. les Commissaires, lorsqu'ils s'opposèrent à ce que nous fussions chargés de police hors l'intérieur de la salle ». S'adressant au Président de l'Assemblée, ils renouvelèrent leur demande d'être en charge de la police extérieure de la salle en arguant de leurs "fonctions honorables qui consistent à annoncer les députations, à avertir les ministres au nom du corps législatif ». Ils entendaient se défendre des critiques émises à leur encontre ${ }^{39}$. Durant la Convention, les huissiers participaient à la sécurité des séances de l'assemblée ; mais, ils restaient sous le contrôle des inspecteurs. Un procès-verbal du Comité des Inspecteurs du 29 messidor an III-17 juillet 1795 fait état de « réformes ordonnées par décret sur la réduction du nombre des employés et charge la troisième section de lui faire un rapport sur les huissiers de la Convention afin qu'il soit statué sur ceux qu'il est indispensable de conserver et ceux qui doivent être remplacés $»^{40}$.

Comme on vient de le voir, le Comité des Inspecteurs avait un rôle multiforme souhaité par la Convention qui avait besoin d'une assistance juridique, technique pour la gestion de l'administration générale de l'Assemblée. Cette diversité se retrouve dans les méthodes de travail du comité.

\section{Le comité des Inspecteurs au travail}

39 Le comité n'était pas seulement un organisme consultatif d'assistance juridique et technique. Il avait aussi un rôle opérationnel et participait à l'activité législative et réglementaire de la Chambre dans la mesure où la Convention concentrait les pouvoirs législatif et exécutif surtout à partir de la disparition notamment du ministère de l'Intérieur, soit le 1er avril 1794.

\section{Participation à l'activité législative de la Convention}

Les frais de mission des représentants de la Convention envoyés dans les départements ou aux armées constituèrent un bon exemple du rôle législatif revenant au Comité des Inspecteurs. Les demandes de remboursement des frais de mission par les représentants de la Convention étaient pour le moins, sinon fantaisistes, du moins insuffisamment motivées, comme ce fut le cas de Barras en juin 1795 et encore davantage de Girot-Pouzol qui demanda 120000 livres, avec pour seul motif de «subvenir aux frais de mission » ${ }^{41}$. Pour remédier à ces incohérences, le Comité des Inspecteurs prit un arrêté le 29 floréal an III-18 mai 1795 pour fixer des règles précises de procédure financière. Ce texte distinguait 
3 catégories de frais de mission: la 1ère catégorie correspondait à des frais qualifiés d'ordinaire pour lesquels aucun justificatif de titre n'était nécessaire. La 2ème catégorie visait les dépenses extraordinaires qui «ne sauraient être allouées que sur pièces justificatives ». Quant à la 3ème catégorie, elle regroupait les dépenses secrètes qui devaient être vérifiées et allouées par le comité qui les ordonnait ${ }^{42}$.

41 Un autre exemple peut être cité. Ainsi, le Comité des Inspecteurs fut confronté aux atteintes à l'ordre public commises dans le Jardin national à la fin du mois de septembre 1793. Les présumés coupables étaient par exemple des marchands, agioteurs, citoyens se livrant à certains délits, manifestants ou « factieux ». Le 28 septembre 1793, le Comité des Inspecteurs s'adressa au ministre de l'Intérieur pour lui demander d'appliquer le décret interdisant aux marchands l'accès au Jardin national. Il enjoignit alors au ministre de faire fermer les ouvertures se trouvant sur la terrasse des Feuillants. Le comité soumit à la Convention un projet de décret interdisant l'accès au Jardin national des établissements de traiteur, limonadiers ou échoppes ${ }^{43}$. Le comité participait bien à l'activité législative de la Convention.

42 Mais, le rôle du Comité des Inspecteurs fut plus souvent administratif ou réglementaire, son activité se rapprochant d'un ministère technique d'autant plus que le dirigisme économique du gouvernement révolutionnaire s'amplifia assez considérablement.

\section{Le Comité des Inspecteurs et la prédominance de son activité administrative et réglementaire}

43 Cette activité était révélatrice des méthodes de travail de ce comité, en quelque sorte au quotidien. On peut d'abord observer une division interne des tâches au sein du comité. Ainsi, en 1794, la 5ème section du Comité des Inspecteurs examina l'état de la tribune construite pour les fêtes nationales dans le Jardin national, au devant du pavillon de l'Unité. Elle constata qu'elle était peu solide, ce qui laissait craindre «quelques évènements ». La 5ème section proposa au Comité des Inspecteurs la démolition de la tribune dans les plus brefs délais ${ }^{44}$.

Le comité faisait souvent appel à ses inspecteurs pour l'établissement de rapports techniques qui facilitaient la prise de décisions. Au milieu de l'année 1793, le Comité des Inspecteurs fut confronté à un problème d'illumination de la salle des séances, en raison de l'énormité de la consommation des fluides, due elle-même aux séances continuelles de nuit des députés et membres des comités. Le Comité des Inspecteurs chargea l'un de ses membres, Robin, «de l'établissement d'un rapport général sur le mode économique d'illuminer la salle et les bureaux de la Convention, sur les économies à apporter à toutes les fournitures de la Convention ». Comme la consommation de chandelles augmentait journellement et que l'approvisionnement « ne peut se faire sans prendre sur les besoins les plus urgents du peuple ", le comité décida, au vu du rapport de Robin, de faire usage de bougies ou d'huile pour éclairer les bureaux et « en surveillant très strictement la consommation et en ne permettant pas qu'on en abuse $»^{45}$.

Ces rapports pouvaient aussi concerner des questions économiquement et politiquement essentielles, comme le recensement des "dépouilles de l'Église». Le Comité des Inspecteurs détenait la responsabilité de la coordination des opérations de vérification et de recollement de ces « dépouilles » qui arrivaient à jet continu des communes de France. Les services administratifs (communes, domaines nationaux) devaient faire parvenir au 
comité les procès-verbaux et récépissés des dépôts de biens, notamment précieux. Les inspecteurs Duval de l'Aube et Herard, nommés par arrêté du comité du 23 brumaire an II-13 novembre 1793, remirent un rapport de mission concernant les opérations d'inventaire des objets du culte, « soit six jours entiers pour faire l'inventaire. Il a fallu en faire faire des extraits doubles, faire le recollement desdits effets, les faire emballer et transporter $»^{46}$.

À la faveur du développement de l'interventionnisme économique durant la Convention, l'action du Comité des Inspecteurs affecta des secteurs clés de l'économie nationale, comme l'Imprimerie nationale et les approvisionnements en matières premières. Le Comité des Inspecteurs utilisa des méthodes qui avaient déjà fait leurs preuves avec l'envoi en province des représentants en mission. En effet, pour le comité, il importait d'être bien informé par exemple «sur les prix pratiqués par les imprimeurs, ceux des administrations des départements et des imprimeries particulières ». D'où l'arrêté pris par le comité le 23 frimaire an II-13 décembre 1793 pour obtenir les renseignements nécessaires ${ }^{47}$. Les inspecteurs Bouchereau et Fiquet remirent un rapport au Comité des Inspecteurs qui, ainsi informé, n'innova pas par rapport au tarif fixé antérieurement. Celui-ci prenait en compte le bénéfice revenant à l'Imprimerie nationale et le remboursement des étoffes (matière première). Le tarif retenu par le comité incorporait le montant des dernières augmentations de salaires accordées aux ouvriers de l'Imprimerie nationale.

47 Cette technique du rapport technique soumis au Comité des Inspecteurs fut également utilisée pour les achats de rames de papier dont avait besoin l'Imprimerie nationale. Ainsi, l'Inspecteur Chabanon fut envoyé dans les départements du Puy-de-Dôme et de la Haute-Vienne pour y acheter des rames de papier. Grâce au rapport remis au comité par cet inspecteur, il apparut que le prix d'achat de la rame de papier négocié à Limoges était de 14 livres (frais de port compris), soit une économie de 3 livres par rame par rapport aux prix d'achat des papiers obtenu dans les départements de l'ancienne Normandie (17 livres par rame $)^{48}$.

Le Comité des Inspecteurs dut aussi intervenir pour trouver des solutions aux conflits sociaux qui apparurent notamment dans la papeterie d'Essonne. Ne pouvant négocier luimême avec les ouvriers, le comité prit un arrêté le 9 fructidor an II-26 août 1794 par lequel il nomma deux de ses inspecteurs qui se rendirent sur place afin de "prendre connaissance des faits, entendre les ouvriers, les concilier, ramener l'ordre et la paix, sinon prendre les mesures de rigueur qu'ils jugeront nécessaires contre les ouvriers qui se refuseraient de reprendre leurs travaux et du tout en rendre compte au Comité $»^{49}$. Ce texte laissait assez peu de latitude aux inspecteurs pour négocier avec les ouvriers en grève d'Essonne. Mais, il n'en fut pas toujours ainsi puisque les commissaires détachés auprès des papeteries mises en réquisition étaient invités à assurer une meilleure concorde sociale entre les fabricants de papiers et leurs ouvriers, comme l'indiquait le règlement des papeteries mises en réquisition pour la fabrication du papier des lois du gouvernement ${ }^{50}$.

D’autres commissaires furent détachés par le Comité des Inspecteurs, non pour régler des conflits sociaux, mais pour contrôler la fabrication du papier dans les manufactures de Courtalin, du Marais et d'Essonne. Ces délégations de pouvoirs consenties à ces commissaires furent définies par un arrêté pris le 19 pluviôse an II-7 février 1794 par le Comité des Inspecteurs. Ceux-ci devaient définir les quantités de papier, le nombre et le travail fourni par les ouvriers, les moyens les plus économiques pour faire venir à Paris le 
papier. Les résultats devaient être certifiés et transmis tous les décadis au Comité des Inspecteurs pour qu'ils prissent les arrêtés nécessités par les circonstances ${ }^{51}$. Ces méthodes de travail instauraient donc un système solide de délégation reposant sur un aller et retour entre les commissaires et le comité.

Le Comité des Inspecteurs n'hésitait pas à s'immiscer dans le processus de fabrication du papier d'impression par les fournisseurs en intervenant auprès de la Commission du commerce et des approvisionnements pour livrer aux fabricants des districts de l'Ouest « la quantité de chiffons qui leur sont nécessaires pour tenir leurs engagements vis-à-vis de la Convention $\|^{52}$. Et lorsque la politique économique se fit moins dirigiste, le Comité des Inspecteurs eut recours à des intermédiaires, c'est-à-dire "des commissionnaires probes et actifs ». Ces intermédiaires étaient " chargés de faire arriver des fabriques dans les magasins et d'expédier ces papiers et de leur accorder pour leurs peines et soins la remise d'usage dans le commerce $»^{53}$. Les mêmes commissionnaires devaient faire appel à des voituriers et les régler pour leurs interventions. Ainsi, le Comité des Inspecteurs, en sa séance du 26 floréal an III-15 mai 1795, précisait que le commissionnaire Muret de Limoges était « responsable des qualités de papier portés sur son état et des quantités qui ne sont pas encore livrées au magasin de la Convention $»^{54}$. Ces activités ainsi concédées à des particuliers ne convainquirent pas le Comité des Inspecteurs de leur efficacité puisqu'il demanda à l'un de ses membres, Couturier, de rédiger un rapport sur cette question. Il apparut, en effet, que le papier n'était pas réellement fabriqué, en dépit de la surveillance exercée par ces commissionnaires. Le contrôle exercé par la seconde section du comité fut jugé plus efficace dans la mesure où ce dernier décida de recruter un garde magasin qui devait vérifier les états des commissionnaires, réceptionner les tableaux des agents nationaux, appliquer les prix aux différentes sortes de papiers, préparer les ordonnances de paiement, confectionner les tableaux de chiffons ${ }^{55}$.

51 Ainsi, les méthodes administratives utilisées par le Comité des Inspecteurs offraient toute une gamme de moyens techniques et juridiques alliant les rapports établis par les Inspecteurs dans une optique hiérarchique après enquête sur place; mais, le comité faisait aussi appel à des personnes privées n'ayant aucun lien de dépendance hiérarchique avec le comité (sans toutefois renoncer au contrôle par les inspecteurs du comité). Cette organisation n'était pas caractérisée par la simplicité ou la souplesse. Il faut préciser que les rapports du Comité des Inspecteurs avec les autres comités n'étaient pas non plus simples. Les raisons de cette complexité méritent une analyse.

\section{Les contacts entre le Comité des Inspecteurs et les autres comités}

52 Ces contacts étaient nécessairement nombreux en raison des frontières souvent assez floues entre comités dont les attributions pouvaient entraîner entre eux des chevauchements de compétence. Certes, l'existence de ce que l'on appelé « les comités de gouvernement » permettait d'établir une hiérarchie entre les comités. Il était évident que pendant la période de juillet 1793 à fin juillet 1794 la prééminence des Comités de salut public et de sûreté générale s'imposait tout particulièrement aux autres comités (le Comité des Inspecteurs, comme aux autres comités). Mais, les relations entre le ministère de l'Intérieur et le Comité des Inspecteurs méritent une attention particulière dans la mesure où ce ministère succédait au ministère de la Maison du roi qui avait jusqu'au début de la Constituante la haute main sur l'administration de l'assemblée. 


\section{Les difficiles relations entre le ministère de l'Intérieur et le Comité des Inspecteurs}

53 Au tout début de l'entrée en fonction de la Convention nationale éclata un conflit de compétence entre le ministère de l'Intérieur à propos de la construction de la nouvelle salle des Tuileries. Le procès-verbal du Comité des inspecteurs, en sa séance du 3 novembre 1792, fait état de la protestation du ministère de l'Intérieur en ce qui concerne le contrôle des travaux de la nouvelle salle des Tuileries. Celui-ci estimait que sa responsabilité en matière de travaux ne saurait «s'étendre sur le tout » et demandait à être seul chargé de la surveillance ou être tout à fait déchargé de cette responsabilité. Le Comité des Inspecteurs répliqua en invoquant le décret de l'Assemblée du 25 octobre 1792 qui attribuait au seul ministère de l'Intérieur l'exécution du plan de la nouvelle salle établi par l'architecte de la Convention, Gisors. La question à l'étude concernait une somme de 200000 livres qui devait être mise à la disposition du ministère de l'Intérieur pour financer les travaux d'aménagement des locaux des comités de la Convention. La solution tarda à venir puisqu'il fallut attendre l'arrêté commun du ministère de l'Intérieur et du Comité des Inspecteurs du 13 nivôse an II-2 janvier 1794 pour que le problème fût réglé dans son principe et d'une manière constructive ${ }^{56}$. Le Comité des Inspecteurs rappela qu'il avait toujours surveillé les travaux d'extension demandés par les comités (de salut public et de sûreté générale). Il ajouta qu'il n'avait pas à se substituer au Comité des finances en donnant son avis sur l'emploi des sommes restant à payer. Le Comité des Inspecteurs se plaça sur un plan purement technique en indiquant les sommes à mettre à la disposition du ministère de l'Intérieur pour soutenir les efforts déployés par les ouvriers pour l'achèvement des travaux. Le comité demeura dans cette posture de contrôleur technique et le ministère de l'Intérieur dans celle de maitre de l'ouvrage. La suppression du ministère de l'Intérieur à partir du 1er avril 1794 évita de nouveaux problèmes. Il en surgit d'autres, notamment avec le Comité de salut public et de sûreté générale.

\section{La subordination du Comité des Inspecteurs au Comité de salut public}

La liaison entre les deux comités fut très fréquente à telle enseigne que le Comité des Inspecteurs fut très vigilant dans ses rapports avec le Comité de salut public. D'où le recours à la technique des arrêtés communs aux deux comités pour donner plus de poids aux demandes formulées par le Comité des Inspecteurs aux autres comités. Par ce moyen, la Commission du commerce et des approvisionnements fut invitée à mettre à la disposition de la Convention tout le bois nécessaire à son chauffage ${ }^{57}$.

Mais, dans d'autres situations, le Comité des Inspecteurs dut assumer seul ses responsabilités en faisant appel à des armes juridiques qui constituaient des prérogatives de puissance publique, comme le droit de réquisition. Ainsi, lorsque le Comité des Inspecteurs décida d'envoyer des fournisseurs privés en Haute Vienne et dans le Puy-deDôme, il leur conféra des pouvoirs de réquisition des papiers destinés à l'Imprimerie nationale, pratique irrégulière dans la mesure où le droit de réquisition devait être conféré à des agents publics ou à des administrations. Le Comité des Inspecteurs sollicita du Comité de salut public son accord sur cette dérogation ${ }^{58}$. La réponse du Comité de salut 
public fut une bonne illustration des relations difficiles qu'il pouvait entretenir avec les comités. Il précisait que le Comité des Inspecteurs détenait, de par l'objet de son institution, "toute l'autorité suffisante pour user des droits de réquisition et de préhension, qu'il pouvait envoyer des commissaires pour exercer et remplir toute autre mission qu'il aurait jugé convenable de donner sur les matières soumises à son administration et que l'intervention du Comité de salut public était inutile». Ce dernier ajouta une critique des méthodes de certains comités, comme celui des Inspecteurs, qui avaient tendance « à prendre l'attache ou l'avis d'autres comités auxquels les matières à délibérer n'auraient point été renvoyées", alors qu' «il importe (que les divers comités) se pénétrassent bien de leur indépendance respective pour remplir dans toute leur étendue les devoirs qui leur étaient imposés ». Après ces remontrances du Comité de salut public, le Comité des Inspecteurs décida d'envoyer en mission un de ses inspecteurs, Chabanon, auquel il donna tous pouvoirs de mettre en réquisition les papiers disponibles chez les fabricants ${ }^{59}$.

56 L'importance politique de certaines questions, comme la préservation du patrimoine national et l'embellissement du Palais et du Jardin national doit être signalée. À cet égard, le Comité des Inspecteurs exécuta les décisions du Comité de salut public. Ce dernier encouragea une politique de sélection des œuvres d'art en vertu d'un décret relatif aux emblèmes et attributs de la royauté qu'il fallait ôter. D'où l'arrêté du Comité des Inspecteurs du 27 juillet relatif à l'enlèvement « des morceaux de sculpture, de peinture en dehors et en dedans du Palais et du Jardin national » considérés comme des emblèmes et des attributs de la royauté qu'il fallait extirper parce que contraires à l'idéal révolutionnaire ${ }^{60}$.

57 Le Comité des Inspecteurs intervint aussi dans le domaine de la protection des lieux de manifestations publiques en appliquant les dispositions du Comité de salut public évidemment concerné par les mesures de police que le Comité des Inspecteurs devait prendre pour assurer la protection de l'ordre public dans l'emprise du Jardin national accessible au public, notamment lors des fêtes célébrées dans cette enceinte. Le Comité de salut public ajouta que des corps de réserve «stationnaires » devaient être disposés dans le Jardin national et qu'aucune patrouille ne devait passer au milieu «de citoyens assemblés ». Pour prévenir toute atteinte à l'ordre public, lors de la fête du 14 prairial an III-2 juin 1795, le Comité des Inspecteurs chargea l'un de ses membres de prendre contact avec les représentants du peuple de la direction de la force armée afin de se concerter au sujet de la consigne donnée pour les différentes entrées du Palais et du Jardin national ${ }^{61}$.

58 Si les relations nouées entre le Comité de salut public et le Comité des Inspecteurs furent caractérisées par une dépendance marquée du second par rapport au premier eu égard aux domaines très sensibles concernés, les rapports entre le Comité de salut public et le Comité de sûreté générale furent plus complexes en raison des attributions de ce dernier (police) qui interféraient avec les prérogatives du Comité des Inspecteurs; ce dernier entendant défendre son pré carré du Palais et du Jardin national.

\section{Les relations difficiles entre le Comité de sûreté générale et le Comité des Inspecteurs}

Le Comité des Inspecteurs intervenait sur toutes les questions intéressant le personnel de la Convention et les représentants du peuple. Par exemple, il s'estimait compétent chaque fois qu'un membre du personnel ou un représentant du peuple faisait l'objet de 
poursuites ou d'arrestation. Par exemple, le Comité des Inspecteurs fut prévenu par une pétition qu'un huissier de la salle avait été emprisonné. Il décida de s'adresser au Comité de sûreté générale pour signaler ce cas en précisant que le service assuré par cet huissier était indispensable. Il demanda si le Comité de sûreté générale était en mesure de prendre une décision et «si dans cette attente, il doit être nommé quelqu'un à sa place » ${ }^{62}$. Dans d'autres circonstances, ce fut le Comité des Inspecteurs qui demanda l'arrestation d'un fusilier, membre du corps des vétérans invalides. La décision fut ordonnée par le Commandant de la Compagnie des invalides. Le procès-verbal contenant la décision et les déclarations étaient transmises au Comité de sûreté générale ${ }^{63}$.

Le cas des représentants du peuple était beaucoup plus délicat, en raison de l'importance des fonctions en cause et aussi de la fréquence des mesures d'arrestation qui frappèrent les élus de la Nation, notamment pendant la Terreur. Le Comité des Inspecteurs était mis au courant de l'arrestation des représentants du peuple, lorsque ceux-ci ou les familles demandaient le versement des indemnités parlementaires pour leur permettre de survivre. C'était le Comité des Inspecteurs qui instruisait les demandes, mais il évitait de prendre seul la décision pour ne pas porter atteinte aux attributions du Comité de sûreté générale qui avait la haute main sur les arrestations, depuis le décret du 23 ventôse an II-13 mars 1794, relatif aux poursuites contre les Indulgents et les Exagérés et la loi du 22 prairial an II-10 juin 1794. Le Comité des Inspecteurs s'adressa au Comité de sûreté générale pour lui demander son avis sur la délivrance d'un mandat en paiement d'une indemnité au représentant du peuple dénommé Peyre, mis en état d'arrestation. La prudence du Comité des Inspecteurs s'expliquait par l'attitude extrêmement sourcilleuse du Comité de sûreté générale quant à l'étendue de ses responsabilités, comme le prouvèrent les évènements du 9 thermidor an II, aux dépens de Robespierre et de son groupe.

61 À partir du 1er prairial an III-20 mai 1795, les représentants du peuple, mis en état d'arrestation, ne pouvaient bénéficier d'indemnités si le décret d'arrestation était postérieur à cette date. La transparence ne constituait pas la règle sur ce point. Le Comité des Inspecteurs se vit dans l'obligation d'écrire au Comité de sûreté générale pour obtenir la transmission de la liste des représentants du peuple privés de ces indemnités. Après thermidor an II, le Comité des Inspecteurs dépêcha un de ses membres au Comité de sûreté générale pour clarifier ce sujet ${ }^{64}$. En cas de doute sur la compétence du Comité de sûreté générale ou du Comité des Inspecteurs, ce dernier se réunit extraordinairement le 15 brumaire an III- 5 novembre 1794 sur un dossier d'arrestation transmis par le Comité de sûreté générale. Le Comité des Inspecteurs se déclara incompétent sur un dossier d'arrestation de deux citoyens et considéra que le Comité de sûreté générale était mieux outillé pour procéder lui-même à des enquêtes, surtout quand il y avait complot, avec évidemment atteinte à l'ordre public ${ }^{65}$.

Les relations étaient dans certains cas très tendues comme ce fut le cas pour des mesures de police engagées à proximité de la Convention. Le Comité de sûreté générale prévint le comité des Inspecteurs que des groupes fréquents à proximité de la Convention paraissaient dirigés par des individus qui voulaient à toute force troubler les séances, soit en s'emparant des tribunes, soit en provoquant des disputes sur les affaires publiques aux environs de la salle. Le Comité de sûreté générale invita le Comité des Inspecteurs «à employer les moyens les plus prompts pour faire cesser ce désordre ${ }^{66} \mathrm{Le}$ ton était pour le moins comminatoire. Mais, la compétence du Comité des Inspecteurs était indiscutable en matière de police dans l'enceinte de la Convention ou à proximité, les agents de police 
requis devant rendre compte au comité "sur le champ" des arrestations et des opérations faites «tant dans les environs du Palais national que dans son enceinte ». Le comité était même habilité à recourir à des mesures militaires. Le Comité des Inspecteurs avait aussi des relations avec d'autres comités, d'une manière assez occasionnelle.

\section{Relations occasionnelles avec d'autres comités}

Le Comité des Inspecteurs eut aussi des relations avec le Comité militaire lorsqu'il fut question de supprimer le corps des militaires invalides, dont l'utilisation était trop onéreuse et l'efficacité insuffisante. Les deux comités proposèrent à la Convention le retrait de la loi du 4 juillet 1793. Les relations avec le Comité des finances étaient aussi occasionnelles, notamment lors des paiements à effectuer des travaux immobiliers. Comme nous l'avons vu précédemment, les relations avec la commission des subsistances et approvisionnements pouvaient être fréquentes en fonction des besoins des réquisitions. Une coordination s'imposait normalement, la prééminence du Comité des Inspecteurs facilitant les relations. Enfin, le Comité des Inspecteurs reçut commande du Comité d'Instruction publique pour une collection complète des bulletins et de leurs suites, des procès-verbaux d'assemblée, des journaux, comme Le Journal de Prud'homme, Le Journal des débats des Jacobins, le Journal de Paris et des Hommes libres.

Le Comité des Inspecteurs était donc bien placé pour participer à l'actualité législative et à la protection de la sécurité de la Convention. Il convient à présent de dresser un bilan de son action.

Le Comité des Inspecteurs vit ses compétences et son rôle politique s'étendre dans de fortes proportions à la faveur de la montée en puissance de la Convention et des deux comités de gouvernement, comme le Comité de salut public et le Comité de sûreté générale. La souplesse dont il fit preuve vis-à-vis du Comité de salut public ne s'expliqua pas par la prédominance du parti de la Montagne par rapport au courant de la Plaine, largement représenté dans le Comité des Inspecteurs. Les fonctions de ce dernier étaient surtout administratives, ce qui le conduisit à exécuter ou à mettre en application les décisions du Comité de salut public, comité évidemment politique par définition. La résistance du Comité des Inspecteurs vis-à-vis du Comité de sûreté générale était plus nette parce qu'il avait des compétences de police circonscrites au périmètre du Palais et $\mathrm{du}$ Jardin national et de leurs environs, ce qui pouvait porter atteinte à la toute puissance du Comité de sûreté générale pleinement compétent en matière de police à Paris et dans tous les départements. En définitive, le Comité des Inspecteurs fut l'exécutant par excellence de la Convention parce qu'il sut défendre avec constance et courage l'exterritorialité de l'Assemblée, ce qui était la meilleure des justifications de sa mission au service du bien public et de la République par delà tous les clivages politiques. 


\section{NOTES}

1. A. CASTALDO, Les méthodes de travail de la Constituante, les techniques délibératives de l'Assemblée nationale 1789-1791, Paris, PUF, 1989, p. 228.

2. A.N., C30, 245, pièce 10 .

3. A.Cohen, Le Comité des Inspecteurs de la salle: Une institution originale de la Convention nationale (1792-1795), Paris, L'Harmattan, 2011, p. 27

4. La somme en jeu était de 200000 livres et le Comité des Inspecteurs désigna deux de ses inspecteurs : Fiquet et Guillemardet pour se concerter avec le ministère des finances pour le déblocage de ces fonds, A.N., D*XXXVc 6, p. 144.

5. Ibid., p. 106 et 107.

6. Séance du Comité des Inspecteurs du 23 mai 1793, A.N., D*XXXVc 6, p. 133.

7. Procès-verbaux du Comité des 26 fructidor an III-12 septembre 1795 et 16 vendémiaire an IV-8 octobre 1795 : un abattement de 10000 livres est pratiqué sur un total de prix de 46236 livres.

8. Séance du Comité des Inspecteurs du 24 septembre 1793, A.N., D*XXXVc 7, folio 11.

9. Le Comité des Inspecteurs, en sa séance du 23 frimaire an III-13 décembre 1794 enjoignit à la locataire de quitter les lieux (séance du comité du prairial an II-23 mai 1794, ibid., 8, folio 29). La locataire obtint une indemnité compensatrice de perte de jouissance.

10. Ibid., folios 106 et 107.

11. Ibid., 8, folio 112 .

12. Séance du Comité des Inspecteurs du 6 brumaire an III-27 octobre 1794, A.N., D*XXXVc8, folios 177 et 178.

13. C.KAWA, Les ronds de cuir en Révolution, Paris, Éditions du CHTS, 1996.

14. Séance du Comité des Inspecteurs du 13 messidor an II-1er juillet 1794, A.N., D*XXXVc 8, folio 55.

15. Arrêté commun du Comité de salut public et du Comité des Inspecteurs du 13 pluviôse an III-1er février 1795, ibid., folio 278.

16. Ibid., 7, folios 41 et 42 .

17. Ibid., folios 13,14 et 43 .

18. C.Kawa, Les ronds de cuir en Révolution, op.cit., p. 144.

19. Séance du Comité des Inspecteurs du 11 nivôse an III-31 décembre 1794, A.N., D*XXXVc 8, folio 239.

20. Séance du Comité des Inspecteurs du 11 nivôse an III-31 décembre 1794, ibid., folios 239 et 240.

21. Séance du Comité des Inspecteurs du 23 frimaire an III-13 décembre 1794, ibid., folio 212.

22. Ibid., 9, folio 139.

23. Ibid., 6, p. 194 et 195.

24. Séance du Comité des Inspecteurs du 6 août 1793, ibid., p. 205 et 206.

25. Séance du Comité des Inspecteurs du 28 messidor-19 juillet 1794, ibid., 8, folio 69.

26. Séance du Comité des Inspecteurs 16 prairial an II-4 juin 1794 et arrêté du même jour, ibid., folios 29 et 30 .

27. Séance du Comité des Inspecteurs du 19 messidor an II-7 juillet 1794, ibid., folio 61.

28. Séance du Comité des Inspecteurs du 26 brumaire an III-16 novembre 1794, ibid., folios 186 à 193.

29. A.N., D*XXXVc 7, folios 29, 30 et 31.

30. Ibid.,9, folios 157 et 158 . 
31. Ibid., 7, folios 78 et 79 .

32. Séance du Comité des Inspecteurs du 16 frimaire an II-6 décembre 1793, ibid., folios 40 et 41.

33. Séance du Comité des inspecteurs du 19 vendémiaire an III-10 octobre 1794, ibid., 8, folio 156.

34. A.N., D*XXXVc 6, p. 52.

35. Ibid., p. 148 et 149.

36. Ibid.,7, folios 15 et 16 .

37. Ibid.,8, folios 25 et 26 .

38. Ibid., folios 227 et 228 .

39. Ibid., C132, 471, pièce 18 .

40. Ibid., D* XXXVc 9, folios 150 et 151.

41. Séance du Comité des Inspecteurs du 16 vendémiaire an IV-8 octobre 1795, ibid.,7, folios 38.

42. Séance du Comité des Inspecteurs du 29 floréal an III-18 mai 1795, ibid., 9, folios 98 et 99.

43. Séance du Comité des Inspecteurs du 4 prairial an III-23 mai 1795, ibid., folio 102.

44. Séance du Comité des Inspecteurs du 16 frimaire an III-7 décembre 1794, ibid., 8, folio 210.

45. Séance du Comité des Inspecteurs du 13 messidor an II-1er juillet 1794, ibid., folio 55.

46. Séance du Comité des Inspecteurs du 19 nivôse an II-8 janvier 1794, ibid., 7, folios 60 et 61.

47. Ibid., folio 44.

48. Séance du Comité des Inspecteurs du 23 pluviôse an II-11février 1794, ibid., folios 73 et 74 .

49. Ibid., 8, folio 106.

50. Séance du Comité des Inspecteurs du 26 vendémiaire an III-17 octobre 1794, ibid., folios 166 à 169.

51. Ibid.,7, folios 70 et 71 .

52. Ibid., 8, folios 127 et 128 .

53. Ibid., folios 226 et 227.

54. Ibid., 9, folios 92 et 93.

55. Séance du Comité des Inspecteurs du 16 germinal an III-5 avril 1795, ibid., folio 46.

56. A.N., D*XXXVc 7, folio 50.

57. Arrêté commun du 13 pluviôse an III-1er fvrier 1795, ibid., 8, folio 278.

58. Séance extraordinaire du Comité des Inspecteurs du 14 frimaire an II-4 décembre 1793, ibid.,7, folio 36.

59. Séance du Comité des Inspecteurs du 16 frimaire an II-6 décembre 1793, ibid., folios 40 et 41.

60. Séance du Comité des Inspecteurs du 27 juillet 1793, ibid., 6, p. 198.

61. Séance du Comité des Inspecteurs du 9 prairial an III-28 mai 1795, ibid., 9, folio 103.

62. Comité des Inspecteurs du 29 frimaire an II-19 décembre 1793, ibid., 7, folio 45.

63. Séance du Comité des Inspecteurs du 26 prairial an II-14 juin 1794, ibid., 8, folio 36.

64. Séance du Comité des Inspecteurs du 13 messidor an III-1er juillet 1795, ibid., 9, folio 133.

65. Ibid., 8, folio 182.

66. Comité des Inspecteurs du 19 brumaire an III-9 novembre 1794, ibid., 8, folio 183.

\section{RÉSUMÉS}

L'origine des inspecteurs de la salle date de la Constituante, lorsqu'il fallut jeter les bases de l'administration de l'assemblée. Les inspecteurs avaient en charge les travaux d'aménagement du Manège des Tuileries en salles et bureaux. Puis, ce fut sous la Convention nationale que le rôle 
des Inspecteurs de la s'étoffa en se structurant en comité. Alors qu'initialement les compétences du comité étaient surtout articulées sur les problèmes immobiliers, de nouvelles attributions incombèrent au Comité des Inspecteurs : administration générale, définition d'une politique d'harmonisation des traitements des employés de la salle et de la plupart des comités. Puis, le comité veilla à la sécurité intérieure et extérieure du Palais et du Jardin national et prit en charge des problèmes plus spécifiquement politiques et économiques, comme le recensement et le tri des biens d'Église, la tutelle de l'Imprimerie nationale (approvisionnements en papiers), le contrôle de l'impression des opinions politiques des représentants du peuple. En définitive, le comité eut un rôle essentiel, tout en étant dépendant du Comité de salut public. Il disparut avec la Convention nationale (26 octobre 1795).

Inspecteurs de la Salle were born under the Constituent Assembly. At that time, Inspectors were in charge of renovation works: the Salle des Manèges in the Tuileries, which was formerly used as a Riding School, had to be converted in rooms and offices. Under the Nartional Convent, Inspecteurs de la Salle transformed into a Committee and were given more and more responsibilities: general administration, most of the committees employees' salary payment, Tuileries' Palace and Gardens security and other political or economical problems. Even if it worked under control of the Committee of Safety Public, this Committee thus played a very important role, until it disappeared with the National Convent on October 26, 1795.

\section{INDEX}

Mots-clés : contrôle politique, ordre public, rapports techniques, réquisitions, tutelle

\section{AUTEUR}

\section{ALAIN COHEN}

Universités de Paris-1 Panthéon-Sorbonne et de Rouen 